\title{
Performance of a Single-Crystal Diamond-Pixel Telescope
}

\author{
R. Hall-Wilton, V. Ryjov \\ CERN, Geneva, Switzerland
}

\section{Pernicka}

Institute of High Energy Physics, Vienna, Austria

\author{
V. Halyo, B. Harrop, A. Hunt, D. Marlow \\ Princeton University, Princeton, NJ, USA
}

\section{E. Bartz, J. Doroshenko, D. Hits; S. Schnetzer, R. Stone,} Rutgers, State University of New Jersey, Piscataway, NJ, USA

\section{W. Bugg, M. Hollingsworth, S. Spanier}

University of Tennessee, Knoxville, TN, USA

\section{W. Johns}

Vanderbilt University, Nashville, TN, USA

\begin{abstract}
We describe the results from a beam test of a telescope consisting of three planes of single-crystal, diamond pixel detectors. This telescope is a prototype for a proposed small-angle luminosity monitor, the Pixel Luminosity Telescope (PLT), for CMS. We recorded the pixel addresses and pulse heights of all pixels over threshold as well as the fast-or signals from all three telescope planes. We present results on the telescope performance including occupancies, pulse heights, fast-or efficiencies and particle tracking. These results show that the PLT design concept is sound and indicate that the project is ready to proceed with the next phase of carrying out a complete system test.
\end{abstract}

9th International Conference on Large Scale Applications and Radiation Hardness of Semiconductor Detectors

30 September - 2 October 2009

Florence, Italy

\footnotetext{
*Speaker.

† corresponding author. E-mail: dmitry.hits@ @ern.ch
} 


\section{Introduction}

The Pixel Luminosity Telescope (PLT) is a proposed dedicated luminosity monitor for CMS based on single-crystal diamond pixel sensors. The PLT is comprised of two arrays of eight smallangle telescopes situated one on each end of CMS. The telescopes consist of three equally-spaced planes of diamond pixel sensors with a total telescope length of $7.5 \mathrm{~cm}$. They are located $5 \mathrm{~cm}$ radially from the beam line at a distance of $1.8 \mathrm{~m}$ from the central collision point. The telescope planes consist of single-crystal diamond sensors with active area of $3.6 \mathrm{~mm} \times 3.8 \mathrm{~mm}$ that are bump bonded to the PSI46v2 CMS pixel readout chip (ROC)[1]. The PLT is designed to provide a high-precision measurement of the bunch-by-bunch relative luminosity at the CMS collision point on a time scale of a few seconds and a stable high-precision measurement of the integrated relative luminosity over the entire lifetime of the CMS experiment. Since there is a large uncertainty on the charged particle multiplicity at small angles in minimum bias events, the PLT is not able to measure the absolute luminosity. The PLT provides a precise and stable measurement of the relative luminosity that will be calibrated with an absolute measurement based on a theoretically well determined process such as $\mathrm{W}$ and $\mathrm{Z}$ boson production.

The primary luminosity measurement of the PLT is based on counting the number of telescopes with three-fold coincidences formed from the fast-or, column-multiplicity signal output by the ROC. The fast-or signal, clocked at the bunch crossing rate of $40 \mathrm{MHz}$, indicates the number of double columns that had pixels over threshold in each bunch crossing. In addition, the full pixel information consisting of the row and column addresses and the pulse heights of all pixels over threshold is readout at a lower rate of a few kHZ. This full pixel readout provides tracking information and is a powerful tool for determining systematic corrections and calibrating pixel efficiencies.

Diamond sensors are crucial for the PLT application since they will operate efficiently with only moderate decrease in signal size over the entire lifetime of CMS[2, 3]. Of equal importance, this radiation hardness does not require that the sensors be cooled. Single-crystal diamond is used for the sensor material rather than polycrystalline diamond since the pulse height distribution of single crystal diamond is large and well separated from zero. To determine the performance of the diamond pixel sensors and the soundness of the PLT design, we carried out a test of a prototype telescope in a $150 \mathrm{GeV} / \mathrm{c} \pi^{+}$beam of the CERN SPS. The primary goals of this test were to determine: the yield of good pixel channels that result from the bump-bonding process; the pulse height response of the diamond sensors for minimum ionizing particles; the fast-or signal efficiency; and the tracking capability of the diamond pixel planes.

\section{Detector Preparation}

The sensors were single-crystal Chemical Vapor Deposition (CVD) diamond with nominal thickness of $500 \mu \mathrm{m}$ and physical area $4.7 \mathrm{~mm} \times 4.7 \mathrm{~mm}$ supplied by Diamond Detectors Ltd. The characteristics of each sensor were studied using a ${ }^{90} \mathrm{Sr}$ beta source. We found that most of the sensors measured achieved full charge collection at an applied field below $0.4 \mathrm{~V} / \mu \mathrm{m}$. For the beam test, a bias voltage of $250 \mathrm{~V}$ was applied to each of the three sensor planes corresponding to approximately $0.5 \mathrm{~V} / \mu \mathrm{m}$. 
Deposition of the pixel electrode pattern on the diamonds and the bump-bonding of the sensors to the pixel ROCs were performed at the Princeton University micro-fabrication laboratory. A $4 \mathrm{~mm}$ $\times 4 \mathrm{~mm} \mathrm{Ti} / \mathrm{W}$ electrode was sputtered on one side of the diamond. On the other side, a pixel pattern was produced. The pattern covered an area of $3.9 \mathrm{~mm} \times 4.0 \mathrm{~mm}$ and consisted of an array of 26 $\times 40$ pixels with pitch of $150 \mu \mathrm{m} \times 100 \mu \mathrm{m}$. Each pixel electrode was $125 \mu \mathrm{m} \times 75 \mu \mathrm{m}$ with $25 \mu \mathrm{m}$ gaps between electrodes. Cylindrical indium bumps with diameters of $15 \mu \mathrm{m}$ and heights of $7 \mu \mathrm{m}$ were evaporated onto the pixel pads on both the ROC and the sensor. The sensors were then bump-bonded to the ROC using a Research Devices MA-8 flip-chip bonder with an opticallycontrolled alignment precision of better than $2 \mu \mathrm{m}$. The electromechanical bond was formed by applying pressure only. The ROC has an array of $52 \times 80$ channels larger in area than the sensors. The diamonds were bonded to columns 13 through 38 and rows 41 through 80 at the center of the top edge of the ROC.

The bonded detectors were mounted onto hybrid boards consisting of flex circuits with a ceramic filled fiberglass backing to form the telescope planes. The hybrid board had a circuit for distributing the sensor bias voltage and a flex pigtail for connecting to a High Density Interconnect (HDI) circuit that forms the backbone of a telescope. After being qualified using a ${ }^{90} \mathrm{Sr}$ source, three detector planes were assembled into a telescope. The HDI circuit was then placed into a carbon fiber support ladder. Figure 1 shows a picture of an assembled telescope. During the test beam, the telescope was inserted into an aluminum cartridge frame pictured in Figure 2. This telescope, which consists of the bump-bonded detectors, the hybrid boards, the HDI and the carbon fiber ladder, is a complete PLT prototype, which will be used in the final CMS installation.

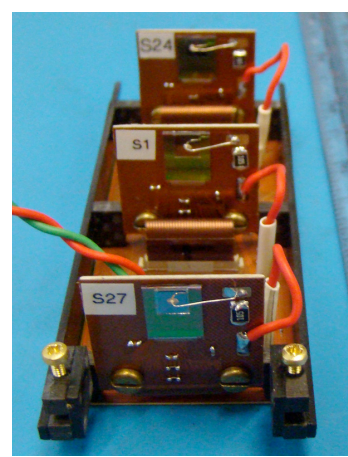

Figure 1: Assembled telescope.

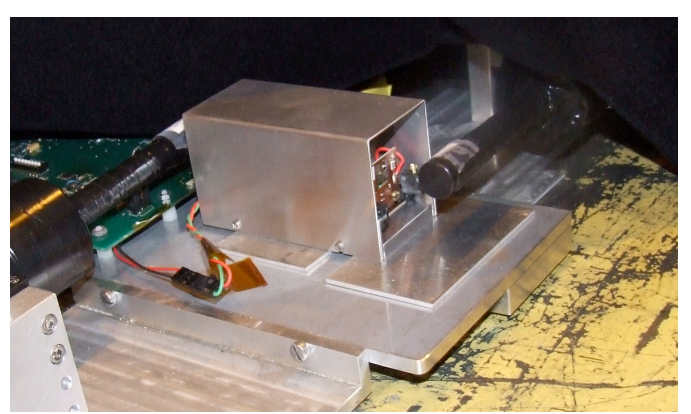

Figure 2: Telescope in test beam with upstream and downstream trigger scintillators.

All columns of all three diamond detector planes were operational except for the five left-most columns of Plane 1 (plane 1, in foreground of Figure 1, is the initial entry point of the beam, left and right defined according to the beam direction). The sensor for this plane had a significant wedge shape varying by about $50 \mu \mathrm{m}$ in thickness from one edge of the diamond to the other. This caused difficulty in the flip-chip bonding, the left-most five columns did not bond. This wedging was due to a temporary defect in the polishing procedure that has since been corrected.

\section{Detector Readout}

A block diagram of the readout scheme used in the beam test is shown in Figure 3. The 
front-end part of the readout chain consisting of the ROC, hybrid board and HDI circuit represents closely the circuitry that will be used in the PLT. The three hybrid board planes, described above, that make up a telescope are connected by pigtails to the HDI, a four-layer flex circuit. The HDI houses a CMS pixel Token Bit Manager (TBM) chip that communicates with the three ROCs in the telescope and orchestrates readout of the full pixel information. This data is output by the TBM onto a single analog line. The HDI also houses a custom PLT driver chip (not shown) that amplifies and outputs the three fast-or signals onto separate analog lines. The HDI also distributes low voltage power and sensor bias voltage to the hybrid boards.

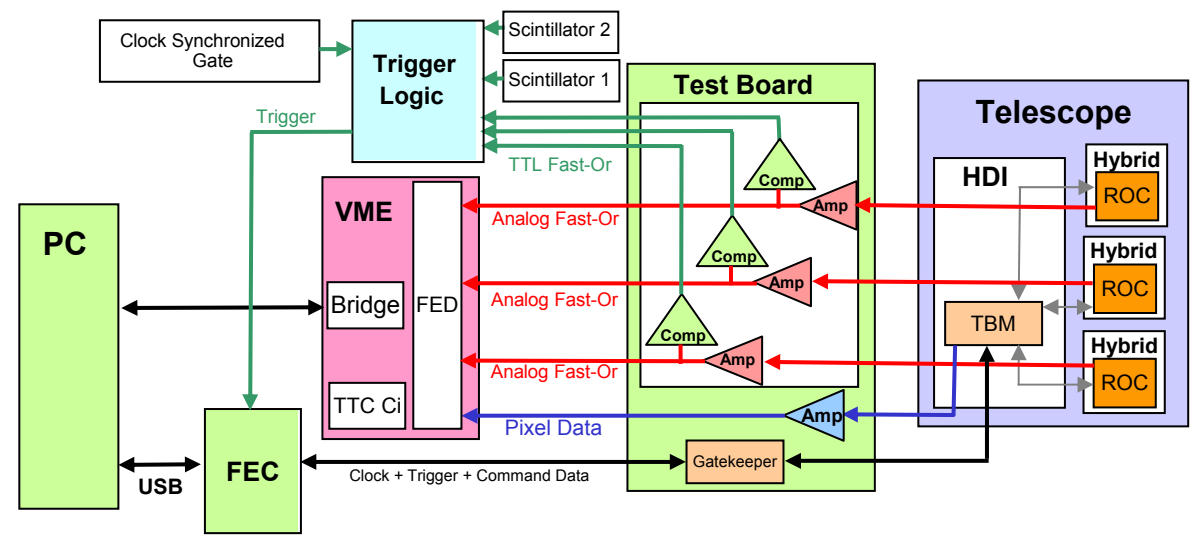

Figure 3: Block diagram of DAQ readout chain.

The HDI was connected to a telescope test stand board. This board, located next to the telescope, produced four amplified, single-ended analog signals from four differential analog signals: one full pixel readout signal from the TBM and three fast-or signals, one from each of the three ROCs. These signals were sent on coaxial cables, approximately $30 \mathrm{~m}$ long, to the control room. The test stand board also output a digital TTL signal for each of the three fast-or signals for use in triggering. A Front End Controller (FEC) test stand board, located in the control room, programed the registers in the TBM and ROCs and set the pixel threshold trim values. It also sent clock and trigger signals to the TBM. The full readout pixel analog signal and the three fast-or signals were input to the FED flash-ADC module. The whole system including ROCs, TBM chip and FED was clocked at $40 \mathrm{MHz}$. A detailed description of the electronic components can be found in [4].

Figure 2 shows the setup in the beam line. Small scintillators each $8 \mathrm{~mm} \times 8 \mathrm{~mm}$ were positioned just upstream and downstream of the telescope. All of the results reported here are based on events triggered by a coincidence of these two scintillators. When a trigger coincidence occurred a signal was sent to the FED via a CMS Timing Trigger and Control Interface $\left(\mathrm{TTC}_{\mathrm{ci}}\right)$ module initiating digitization. To have a wide time view of the event, the digitization started three clock periods before the event and continued for 960 clock periods.

Before taking data the pixel thresholds were lowered as much as possible. This was a multiplestep procedure that involved adjusting three DAC settings in the ROC: the overall coarse threshold, the trimming range around this setting, and the trimmed threshold for each individual pixel. The pixel thresholds achieved were in the range of 2,500 to 4,500 electrons as shown below in the section on the pulse height distribution. 
The telescopes planes were calibrated using the built-in pulsing capability of the ROC. Internally the ROC could be programmed to deposit known amounts of charge into selected pixel channels. The input charge was plotted vs. the FED ADC value and fit to a second-order polynomial to obtain a mapping from ADC count to pulse height in electrons.

\section{Results}

\subsection{Plane Occupancies and Pixel Yield}

Figure 4 shows the occupancy of each of the three telescope planes for events triggered by a coincidence of the two $8 \mathrm{~mm} \times 8 \mathrm{~mm}$ scintillators. The area of each pixel box in the figure is proportional to the number of hits on the pixel. As noted above, the first five columns of Plane 1 were disabled because of bonding problems caused by the wedge shape of this sensor. Due to several noisy pixels, the rightmost column of Plane 1 was also disabled. All of the rest of the columns in all three planes were enabled. Because of limited beam time, we were not able to fully align the telescope and the scintillators with the beam. As a result, the leftmost columns of the telescope planes were not covered by the scintillators, as can be seen in the figure. The scintillator coverage varied by plus or minus one column among the three planes due to small relative misalignment of the planes. The outermost rows and columns collect charge from particles over a region extending outside of the pixelized region and, therefore, have more hits than other rows and columns. In the PLT, these outermost rows and columns will be masked to define a sharp fiducial region given by the boundary between the outermost rows (columns) and their neighboring row (column).
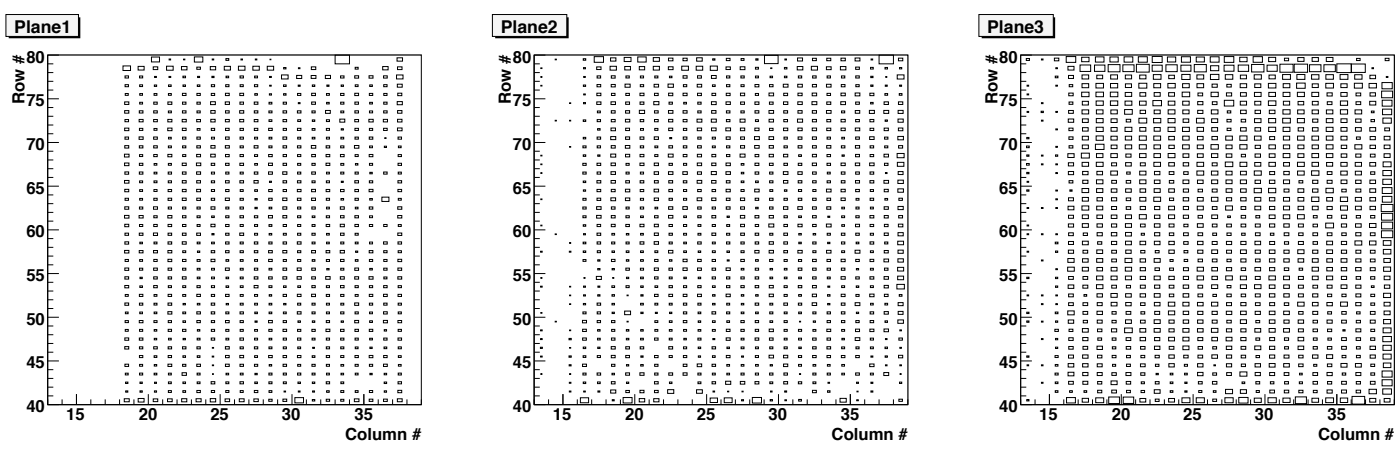

Figure 4: Occupancy map for each of the three telescope planes. Area of each pixel box is proportional to the number of hits on the pixel.

In all three planes, pixels in Row 78 also tend to have a larger number of hits than other rows. This is not currently understood. The six empty pixels in Column 37 of Plane 1 were noisy and were masked off. Except for shadowed and disabled columns, nearly all pixels were active. The number of pixels without any hits are 1.8\%, 2.2\% and $0.1 \%$ for Planes 1, 2 and 3, respectively, indicating that, for these planes, the yield of good pixel bump connections was $98 \%$ or better.

\subsection{Tracking}

To reconstruct tracks in the telescope, we first found the clusters associated with particle hits in each plane. For our analysis, clusters in which three or more pixels were aligned in either a 
row or column were excluded. The cluster position was calculated as the average of the positions of its constituent pixels weighted by their collected charge. Figure 5 shows a sample of tracks reconstructed from the cluster positions.

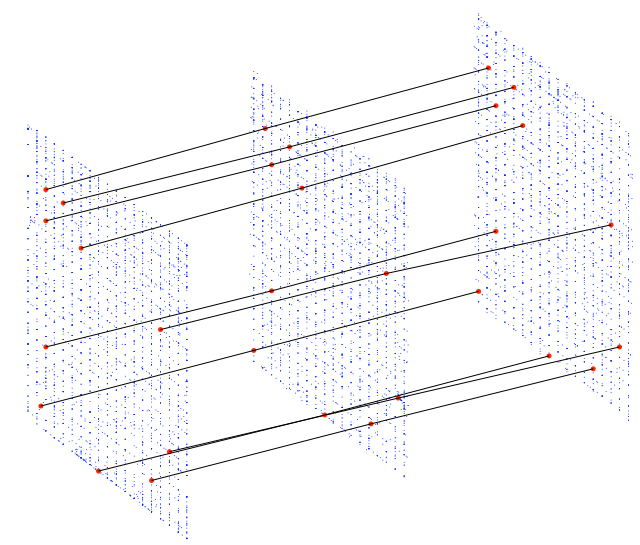

Figure 5: A sampling of tracks, each track corresponds to separate event, reconstructed in the telescope.

The tracking information allowed us to readily determine the relative alignment of the three planes. Using the difference between the hit cluster position in Plane 2 and the average of the hit cluster positions in Planes 1 and 3, we determined the relative offset of Plane 2 with respect to Planes 1 and 3. This offset was $25 \mu \mathrm{m} \pm 5 \mu \mathrm{m}$ in the column direction and $146 \mu \mathrm{m} \pm 3 \mu \mathrm{m}$ in the row direction. In addition, the difference of the hit cluster positions in two planes vs. the hit cluster position in the orthogonal direction allowed us to determine the relative rotation of the three planes about the beam direction. The rotation of Plane 2 was 0.6 degrees with respect to Planes 1 and 3. This alignment of the telescope planes was done readily with only a small amount of data indicating that the alignment of the PLT will be straightforward and quick.

\subsection{Pulse Height Distributions}

For determining pulse height distributions, we defined an acceptance region in each of the three planes such that if a beam particle were incident on this region in two of the planes it was certain to also be incident on the enabled area of the third plane. Figure 6 shows the summed pulse height distributions for each of the three planes where for a given plane a cluster within the acceptance region of the other two planes was required. We also required that there be one and only one cluster in each of these two planes. The pulse height plotted is the sum over all pixels within the hit cluster. The most probable signal is approximately 16,000 electrons for Plane 1 and approximately 18,500 electrons for Planes 2 and 3. There is a 10\% systematic uncertainty in calibration from plane to plane. The most probable pulse height in Plane 1 is about $15 \%$ lower than that for Planes 2 and 3. This is largely due to the fact that the average thickness of the sensor for this plane was less than that of the other two. Planes 2 and 3 were $499 \mu \mathrm{m}$ and $496 \mu \mathrm{m}$, respectively while Plane 1 was wedge-shaped and had an average thickness of $457 \mu \mathrm{m}$ with a variation of $50 \mu \mathrm{m}$ from one side to the other. The small bump in the pulse height distribution of Plane 2 at around 5,000 electrons is not yet fully understood but seems to be due to about fifteen pixels that have anomalously low pulse heights. 
Plane 1

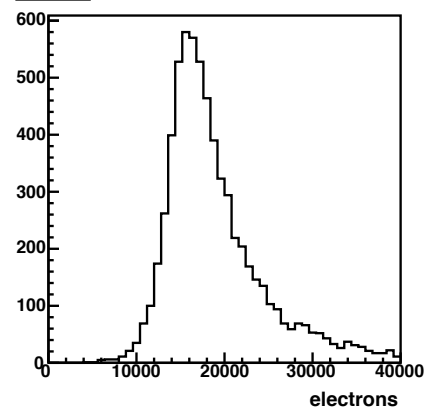

\section{Plane 2}

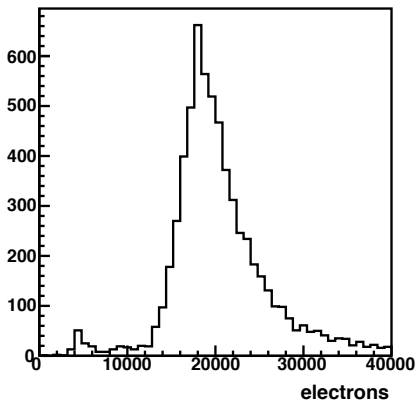

Plane 3

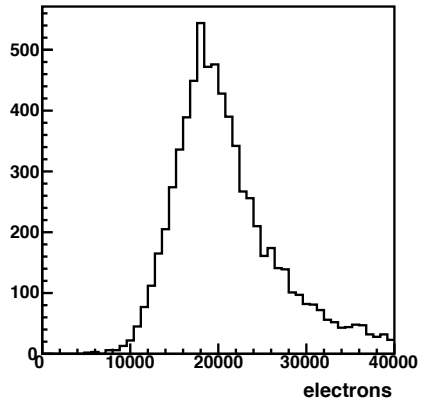

Figure 6: Distribution of the summed pulse height for each of the three telescope planes.
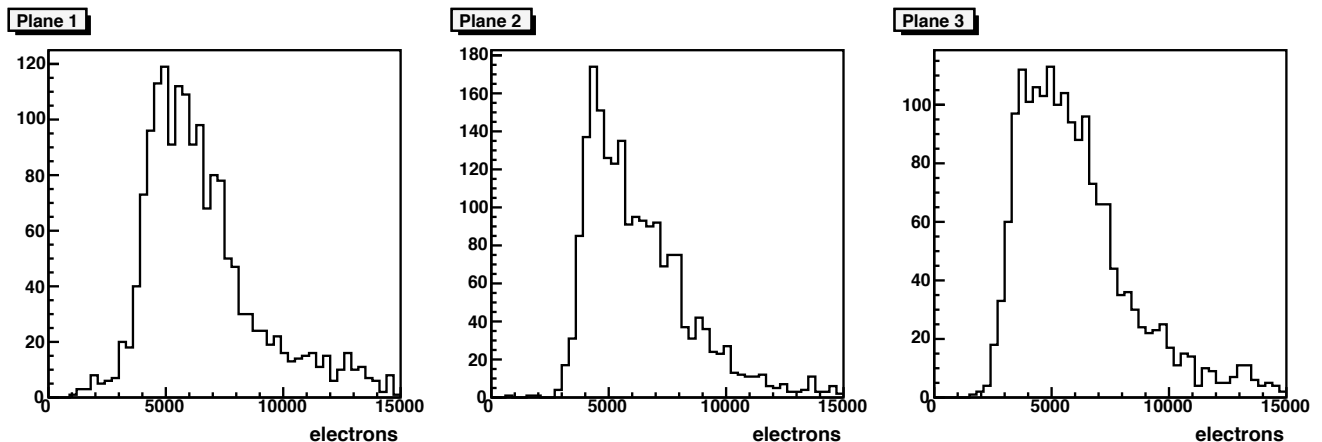

Figure 7: Pulse height distributions for the pixel with lowest pulse height for events in which two pixels are over threshold.

We can obtain a measure of the pixel threshold settings by examining the turn on of the distribution for low pulse heights. Figure 7 shows the pulse height distributions of the lowest pulse height pixel for the case of two-pixel clusters. These distributions indicate that the threshold turn on is 3,500 to 4,500 electrons, 3,000 to 4,000 electrons and 2,500 to 3,500 electrons for Planes 1 , 2 and 3 , respectively.

\subsection{Fast-Or Efficiencies}

The fast-or signals form the basis for the primary luminosity measurement of the PLT. Understanding these fast-or signals and measuring their efficiency is key to establishing the performance of the PLT. For determining the fast-or efficiencies, we imposed the same requirements as for the pulse height measurement above. The fast-or efficiencies are shown in the Table 1 below. The correction due to accidental firing of the fast-or signal is negligible. In the nine clock pulses occurring between $2.50 \mu \mathrm{s}$ and $2.75 \mu \mathrm{s}$ after a triggered event, there were 87 fast-or's out of 100,000 events giving a $0.03 \%$ probability for an accidental fast-or in a three clock period.

In a given event, the presence of a fast-or signal depends on whether or not the pixel with maximum pulse height is above threshold. Figure 8 shows this maximum pulse height distribution for each of the three planes. The lower edge of these distributions is above the measured thresholds. 


\begin{tabular}{|c|c|c|c|}
\hline & Plane 1 & Plane 2 & Plane 3 \\
\hline Total events & 7,146 & 9,049 & 7,814 \\
Events with no fast-or & 53 & 37 & 7 \\
Efficiency & $99.3 \%$ & $99.6 \%$ & $99.9 \%$ \\
\hline
\end{tabular}

Table 1: Fast-or percentages and efficiencies.
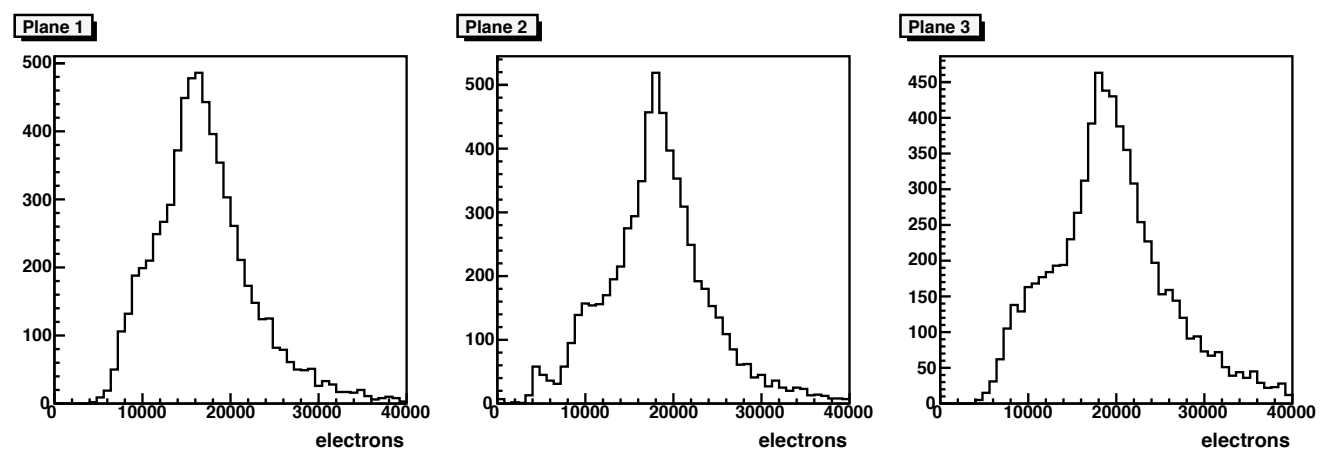

Figure 8: Distribution of pulse height of the pixel with maximum pulse height for each of the three telescope planes.

\section{Conclusions}

We have completed a preliminary analysis of beam test data of a prototype PLT telescope. The fraction of active pixel channels is high, $98 \%$ or more, in all three planes. The pulse height distribution for the high energy beam pions has its most probable value at about 18,000 electrons. The lower edge of the pulse height distributions, for the pixels with max pulse height, is above the pixel threshold range of 2,500 to 4,500 electrons. Thus, the efficiency of the fast-or signals that form the basis of the primary PLT luminosity measurement is high, greater than $99 \%$ for all three planes. Clear and well defined tracks are readily reconstructed in the telescope. These results show that the PLT design concept is sound and that the project is ready to proceed to the next phase, a complete system test of a set of PLT telescopes.

\section{References}

[1] M. Barbero et al., "Design and test of the CMS pixel readout chip," Nucl. Instrum. Meth. A 517, 349 (2004).

[2] W. Adam et al., "Radiation hard diamond sensors for future tracking applications," Nucl. Instrum. Meth. A 565, 278 (2006).

[3] M. Barbero et al., "Development of Diamond Tracking Detectors for High Luminosity Experiments at the LHC." CERN LHCC-Report CERN/LHCC 2008-005, LHCC-RD-016, 2008.

[4] D. Kotlinski et al., "The control and readout systems of the CMS pixel barrel detector," Nucl. Instrum. Meth. A 565, 73 (2006). 